\title{
Efeito do produto comercial valio a base de óleo de laranja na qualidade da aplicação
}

\author{
Effect of commercial product worth orange oil base on application quality \\ Efecto del producto comercial a base de aceite de naranja sobre la calidad de la aplicación
}

Recebido: 19/01/2022 | Revisado: 23/01/2022 | Aceito: 27/01/2022 | Publicado: 28/01/2022

Wendson Soares da Silva Cavalcante

ORCID: https://orcid.org/0000-0002-5224-5486

Universidade de Rio Verde, Brasil

E-mail: wendsonbfsoarescvt@gmail.com

Nelmício Furtado da Silva

ORCID: https://orcid.org/0000-0001-7055-8075

Universidade de Rio Verde, Brasil

E-mail: nelmiciofurtado@gmail.com

Marconi Batista Teixeira

ORCID: https://orcid.org/0000-0002-0152-256X Instituto Federal de Educação, Ciência e Tecnologia Goiano, Brasil

E-mail: marconi.teixeira@ifgoiano.edu.br

Giacomo Zanotto Neto

ORCID: https://orcid.org/0000-0001-8167-5066 Instituto Federal de Educação, Ciência e Tecnologia Goiano, Brasil E-mail: giacomozn@gmail.com

Estevão Rodrigues

ORCID: https://orcid.org/0000-0002-5224-5486 MRE - Agro Pesquisa, Brasil E-mail: estevao.agro@gmail.com

Fernando Rodrigues Cabral Filho

ORCID: https://orcid.org/0000-0002-5090-5946 Instituto Federal de Educação, Ciência e Tecnologia Goiano, Brasil E-mail: fernandorcfilho@hotmail.com

Fernando Rezende Corrêa

ORCID: https://orcid.org/0000-0001-7110-3611 UniBRAS Faculdade Rio Verde, Brasil E-mail: fernandorvcorrea@gmail.com

\begin{abstract}
Resumo
O presente trabalho teve como objetivo comparar o produto comercial Valio ${ }^{\circledR}$ a base de óleo de laranja com óleo mineral e adjuvante na deposição de gotas de pulverização de produtos químicos nos alvos desejados na aplicação terrestre. $\mathrm{O}$ teste foi realizado em condições de campo, utilizou-se a cultura do feijoeiro comum no municio de Rio Verde, Goiás, na estação experimental MRE Agropesquisa Ltda, localizada nas coordenadas geográficas $\left(17^{\circ} 55^{\prime} 23.68^{\prime \prime S}\right.$ e $\left.51^{\circ} 8^{\prime} 45.32^{\prime \prime O}\right)$. Para a deposição de gotas, foram colocados três coletores de papel hidrossensível distribuídos transversalmente à linha de aplicação ao longo da barra de pulverização. O delineamento foi inteiramente casualizado com quatro repetições, considerando o controle como $0 \mathrm{~mL} \mathrm{ha}^{-1}$ de produto comercial na calda. Os papeis hidrossensíveis foram escaneados e analisados com o auxílio do software de análise de deposição de agrotóxicos DepositScan ${ }^{\circledR}$ e depois tabulados no Excel $^{\circledR}$. Foram definidos o tamanho de gotas, número de gotas, cobertura e volume para todos os tratamentos nas diferentes posições de coleta. O produto comercial Valio ${ }^{\circledR}$ (óleo de laranja) teve um comportamento satisfatório na qualidade da aplicação.
\end{abstract}

Palavras-chave: Adjuvante; Deposição de gotas; Terço inferior; Tecnologia de aplicação; Pulverização.

\begin{abstract}
This study aimed to compare the commercial product Valio® based on orange oil with mineral oil and adjuvant in the deposition of spray droplets of chemical products on the desired targets in land application. The test was carried out under field conditions, using the common bean crop in the municipality of Rio Verde, Goiás, at the experimental station MRE Agropesquisa Ltda, located at geographic coordinates (1755'23.68"S and 51 ${ }^{\circ} 45.32$ "THE). For the deposition of drops, three water-sensitive paper collectors were placed transversally to the application line along the spray bar. The design was completely randomized with four replications, considering the control as $0 \mathrm{~mL}$ ha-1 of commercial product in the syrup. The water-sensitive papers were scanned and analyzed using the DepositScan ${ }^{\circledR}$ pesticide deposition analysis software and then tabulated in Excel@. Drop size, drop number, coverage and volume were defined for all treatments in different collection positions. The commercial product Valio® (orange oil) had a satisfactory performance in terms of application quality.
\end{abstract}

Keywords: Adjuvant; Droplet deposition; Lower third; Application technology; Pulverization. 


\section{Resumen}

El presente trabajo tuvo como objetivo comparar el producto comercial Valio $₫$ a base de aceite de naranja con aceite mineral y coadyuvante en la deposición de gotas de aspersión de productos químicos sobre blancos deseados en aplicación terrestre. La prueba se realizó en condiciones de campo, utilizando el cultivo de frijol común en el municipio de Rio Verde, Goiás, en la estación experimental MRE Agropesquisa Ltda, ubicada en las coordenadas geográficas $\left(17^{\circ} 55^{\prime} 23.68\right.$ "S y 51 8'45.32 " EL). Para la deposición de gotas se colocaron tres colectores de papel hidrosensible transversalmente a la línea de aplicación a lo largo de la barra de aspersión. El diseño fue completamente al azar con cuatro repeticiones, considerando como testigo $0 \mathrm{~mL}$ ha- 1 de producto comercial en la aspersión. Los papeles sensibles al agua se escanearon y analizaron usando el software de análisis de deposición de pesticidas DepositScan ${ }^{\circledR}$ y luego se tabularon en Excel®. Se definió el tamaño de gota, el número de gotas, la cobertura y el volumen para todos los tratamientos en diferentes posiciones de recolección. El producto comercial Valio® (aceite de naranja) tuvo un desempeño satisfactorio en cuanto a la calidad de aplicación.

Palabras clave: Auxiliar; Deposición de gotitas; Tercio inferior; Tecnología de aplicación; Pulverización.

\section{Introdução}

A tecnologia de aplicação utilizada para a pulverização da cultura é um dos principais fatores para o sucesso do uso adequado de defensivos agrícolas. Um dos principais critérios a ser considerado são as características das gotas geradas pelo pulverizador, medidas junto ao alvo, no caso, em geral as folhas na altura desejada na planta (Jadoski et al., 2009).

O volume ideal é aquele que gera gotas corretas e adequadas para cada tipo de aplicação, proporciona boa deposição sobre as plantas, excelente penetração dentro do dossel, alta eficiência e minimização de perdas (Tinos et al., 2010).

Para vencer estas barreiras das plantas à penetração dos defensivos, são utilizadas substâncias inertes, denominadas aditivos ou adjuvantes, capazes de modificar a atividade dos produtos aplicados e as características da pulverização. Estes produtos podem ser acrescentados à formulação dos agrotóxicos pelas empresas fabricantes, ou ser adicionados à calda no momento da pulverização (Theisen \& Ruedeil, 2004).

Segundo Vargas e Roman (2006), os adjuvantes são divididos em dois grupos: os modificadores das propriedades de superfície dos líquidos (surfatantes, espalhante, umectante, detergentes, dispersantes e aderentes, entre outros) e os aditivos (óleo mineral ou vegetal, sulfato de amônio e uréia, entre outros) que afetam a absorção devido à sua ação direta sobre a cutícula.

Dentre os grupos de adjuvantes, os surfatantes são os mais utilizados, pois interferem positivamente na emulsão, dispersão, espalhamento, molhamento e, principalmente, na eficiência de controle de pragas e doenças dos produtos fitossanitários (Lenz et al., 2011). Os mesmos se enquadram em um grupo de adjuvantes com maior representatividade comercial e maior número de pesquisas (Iost \& Raetano, 2011).

Juliatti (2035) comenta que no centro-oeste o período para realizar as aplicações é curto, uma vez que geralmente a temperatura do ar, velocidade do vento e umidade relativa do ar não são adequadas para as pulverizações. Condições adequadas para as pulverizações geralmente são observadas apenas no início da manhã e final da tarde. Sendo assim, quando se dispõe de maior rendimento operacional das máquinas, têm-se também melhor aproveitamento das boas condições climáticas para as pulverizações.

Tormen et al. (2012), avaliando a deposição de gotas promovida por diferentes pontas de pulverização, apontaram a eficiência da penetração de gotas finas, indicado pelos maiores depósitos nos terços médio e inferior da cultura da soja, no entanto, os autores ressaltam que o índice de área foliar da cultura deve ser levado em conta para ser feita a escolha do tamanho de gotas.

Recentemente, desenvolveu-se adjuvantes naturais baseados em óleos essenciais extraídos da casca de laranjas (Citrus spp. L.), no entanto, há carência de informações sobre sua eficiência (Souza et al., 2014; Coradini et al., 2016; Zampiroli et al., 2019). Diante do exposto o presente trabalho teve como objetivo comparar o produto comercial Valio a base de óleo de laranja 
com óleo mineral e adjuvante na deposição de gotas de pulverização de produtos químicos nos alvos desejados na aplicação terrestre.

\section{Metodologia}

O teste foi realizado em condições de campo, utilizou-se a cultura do feijoeiro comum (Phaseolus vulgaris L.) na fase de enchimento de grãos, simulando condições de difícil penetração de gotas dentro do dossel das plantas tanto para controle de pragas como doenças. O experimento foi realizado no Município de Rio Verde, Goiás, na estação experimental MRE

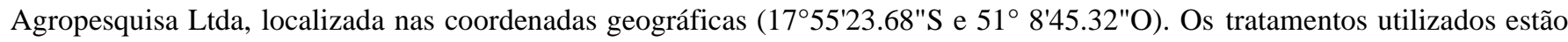
descritos na Tabela 1.

Tabela 1. Tratamentos utilizados, Rio Verde - GO.

\begin{tabular}{cccc}
\hline Tratamento & Produto comercial $^{1}$ & $\begin{array}{c}\text { Ingrediente ativo } \\
\text { (i.a.) }\end{array}$ & $\begin{array}{c}\text { Dose } \\
\left(\mathrm{L} \mathrm{ha}^{-1}\right)\end{array}$ \\
\hline T1 & Valio $^{\circledR}$ & Óleo de laranja & 0,05 \\
T2 & Valio $^{\circledR}$ & Óleo de laranja & 0,10 \\
T3 & Valio $^{\circledR}$ & Óleo de laranja & 0,15 \\
T4 & Valio $^{\circledR}$ & Óleo de laranja & 0,20 \\
T5 & Valio $^{\circledR}$ & Óleo de laranja & 0,25 \\
T6 & Vortexy $^{\circledR}$ & Adjuvante & 0,05 \\
T7 & Aureo & Óleo mineral & 0,25 \\
T8 & Controle $^{\circledR}$ & Controle & 0,00 \\
\hline
\end{tabular}

\footnotetext{
${ }^{1}$ Os produtos comerciais testados "valio e vortexy" foram cedidos e permitida a variação de dose e divulgação do nome comercial pela empresa Tecno Nutrição Vegetal e Biotecnologia Ltda. Fonte: Autores.
}

Foram utilizados os produtos comerciais (Vortexy e Aureo) dentro das respectivas doses de recomendação de bula e o produto comercial (Valio) em diferentes doses. A calda contendo os produtos comerciais nas diferentes doses foi preparada no momento da aplicação em garrafas de $2 \mathrm{~L}$. Foi calculado a quantidade de produto de acordo com os tratamentos e em função do volume de calda. A água utilizada para preparo da calda foi oriunda da torneira, sistema de abastecimento da cidade de Rio Verde - GO, foi mensurado um valor de $\mathrm{pH}$ 7,5 no momento de preparo da calda.

A aplicação foi realizada utilizando um pulverizador costal com pressurização por $\mathrm{CO}_{2}$, munido de barra de $2 \mathrm{~m}$, contendo quatro pontas de pulverização do tipo TT 110-02 (0,50 m entre pontas), aplicando volume de calda equivalente a 120 $\mathrm{L} \mathrm{ha}^{-1}$ (Cavalcante et al., 2020). As condições ambientais nos momentos das aplicações foram monitoradas para obter uma condição favorável de temperatura, UR e velocidade do vento por um Anemômetro marca Kestrel ${ }^{\circledR}$, modelo 3000. As aplicações foram realizadas entre 8:00 e 10:00 horas, período que foi possível reunir as melhores condições climáticas para as aplicações. As condições ambientais médias nos momentos das aplicações foram: temperatura $26^{\circ} \mathrm{C}$, UR $65 \%$ e velocidade do vento $4,0 \mathrm{~m} \mathrm{~s}^{-1}$.

Para a deposição de gotas, foram colocados três coletores de papel hidrossensível distribuídos transversalmente à linha de aplicação ao longo da barra de pulverização. Estes coletores foram espaçados entre si de $3 \mathrm{~m}$. A linha de coletor (papel hidrossensível) foi avaliada $3 \mathrm{~m}$ após o início da pulverização, para garantir a estabilização da pressão do pulverizador e da velocidade de deslocamento do aplicador. Foram utilizados papeis hidrossensíveis (76 x 26 mm-Syngenta/Micron) fixados em folhas do terço superior, médio e inferior das plantas. Posteriormente a aplicação os papeis foram identificados para análise.

$\mathrm{O}$ delineamento foi inteiramente casualizado com quatro repetições, considerando o controle como $0 \mathrm{~mL} \mathrm{ha}^{-1} \mathrm{de}^{-}$ produto comercial na calda. Os papeis hidrossensíveis foram escaneados e analisados com o auxílio do software de análise de deposição de agrotóxicos DepositScan ${ }^{\circledR}$ (Zhu et al., 2011) e depois tabulados no Excel $^{\circledR}$. Foram definidos o tamanho de gotas, número de gotas, cobertura e volume para todos os tratamentos nas diferentes posições de coleta. 
Os dados foram submetidos a análise de variância $(p<0,05)$ e em caso de significância foram submetidos ao teste de média (Tukey $p<0,05$ ), utilizando o software estatístico SISVAR (Ferreira, 2011).

\section{Resultados e Discussão}

Na Tabela 2, observa-se as médias obtidas em relação ao terço da planta onde foram coletados os valores de diâmetro de gotas, cobertura, número de gotas, volume e área em função dos tratamentos.

Tabela 2. Valores de diâmetro de gotas, cobertura, número de gotas, volume e área em função dos tratamentos, Rio Verde GO.

\begin{tabular}{|c|c|c|c|c|c|c|c|c|c|}
\hline \multirow{2}{*}{ Tratamento } & \multirow{2}{*}{$\begin{array}{l}\text { Terço } \\
\text { Planta }\end{array}$} & \multicolumn{3}{|c|}{ Diâmetro de gotas (\%) } & \multirow{2}{*}{$\begin{array}{c}\text { Cobertura } \\
\%\end{array}$} & \multicolumn{2}{|c|}{ Gotas } & \multirow{2}{*}{$\begin{array}{l}\text { Volume } \\
\mu \mathrm{L} \mathrm{cm}^{-2}\end{array}$} & \multirow{2}{*}{$\begin{array}{c}\text { Área } \\
\mathrm{cm}^{2} \\
\end{array}$} \\
\hline & & $1 \mu \mathrm{m}$ & $5 \mu \mathrm{m}$ & $9 \mu \mathrm{m}$ & & $\mathrm{cm}^{2}$ & totais & & \\
\hline $\mathrm{T} 1$ & $S$ & 12,27 & 33,24 & 54,49 & 35,68 & 59,49 & 302,00 & 3,00 & 9,98 \\
\hline Valio & M & 16,33 & 31,50 & 52,17 & 13,76 & 38,43 & 202,00 & 0,52 & 10,28 \\
\hline$\left(50 \mathrm{~mL} \mathrm{ha}^{-1}\right)$ & I & 14,54 & 31,59 & 53,88 & 8,25 & 32,64 & 177,50 & 0,30 & 10,37 \\
\hline $\mathrm{T} 2$ & $\mathrm{~S}$ & 13,01 & 32,34 & 54,65 & 30,21 & 57,85 & 364,50 & 1,14 & 11,16 \\
\hline Valio & M & 17,43 & 29,41 & 53,15 & 16,09 & 34,02 & 216,50 & 0,33 & 11,26 \\
\hline$\left(100 \mathrm{~mL} \mathrm{ha}^{-1}\right)$ & $\mathrm{I}$ & 14,81 & 32,34 & 52,85 & 11,36 & 38,37 & 228,00 & 0,37 & 10,87 \\
\hline $\mathrm{T} 3$ & $\mathrm{~S}$ & 14,73 & 33,35 & 51,92 & 29,16 & 53,40 & 343,50 & 1,34 & 11,26 \\
\hline Valio & M & 15,57 & 31,54 & 52,90 & 17,99 & 64,47 & 370,00 & 0,63 & 10,67 \\
\hline$\left(150 \mathrm{~mL} \mathrm{ha}^{-1}\right)$ & I & 14,59 & 29,93 & 55,47 & 12,57 & 35,48 & 204,50 & 0,51 & 10,67 \\
\hline $\mathrm{T} 4$ & $S$ & 14,29 & 36,45 & 49,26 & 35,17 & 52,46 & 312,50 & 2,03 & 10,87 \\
\hline Valio & M & 14,44 & 35,01 & 50,55 & 23,48 & 54,31 & 305,50 & 0,96 & 10,57 \\
\hline$\left(200 \mathrm{~mL} \mathrm{ha}^{-1}\right)$ & $\mathrm{I}$ & 16,69 & 30,66 & 52,64 & 14,46 & 50,66 & 307,00 & 0,47 & 10,97 \\
\hline T5 & $S$ & 13,83 & 35,33 & 50,83 & 37,63 & 42,10 & 237,13 & 2,68 & 10,57 \\
\hline Valio & M & 15,27 & 31,84 & 52,89 & 22,60 & 40,44 & 244,38 & 1,11 & 10,97 \\
\hline$\left(250 \mathrm{~mL} \mathrm{ha}^{-1}\right)$ & $\mathrm{I}$ & 17,13 & 30,88 & 51,98 & 13,81 & 58,04 & 343,75 & 0,41 & 10,77 \\
\hline T6 & $S$ & 13,40 & 35,36 & 51,24 & 38,87 & 66,60 & 90,50 & 7,68 & 10,57 \\
\hline Vortexy & M & 16,12 & 32,71 & 51,17 & 18,55 & 98,27 & 215,00 & 0,49 & 11,07 \\
\hline$\left(50 \mathrm{~mL} \mathrm{ha}^{-1}\right)$ & I & 17,07 & 30,26 & 52,67 & 10,99 & 73,65 & 149,50 & 0,20 & 10,37 \\
\hline $\mathrm{T} 7$ & $S$ & 10,78 & 34,31 & 54,92 & 48,87 & 79,30 & 217,00 & 3,36 & 10,47 \\
\hline Aureo & M & 13,92 & 28,30 & 57,79 & 14,91 & 83,33 & 291,50 & 0,70 & 10,68 \\
\hline$\left(60 \mathrm{~mL} \mathrm{ha}^{-1}\right)$ & $\mathrm{I}$ & 14,78 & 30,23 & 54,99 & 7,81 & 48,90 & 115,50 & 0,18 & 10,28 \\
\hline $\mathrm{T} 8$ & $S$ & 11,86 & 34,62 & 53,52 & 33,39 & 60,58 & 333,00 & 2,08 & 10,47 \\
\hline Controle & M & 15,81 & 30,10 & 54,09 & 17,39 & 53,73 & 302,00 & 0,65 & 10,57 \\
\hline$\left(0 \mathrm{~mL} \mathrm{ha}^{-1}\right)$ & I & 13,40 & 28,59 & 58,01 & 6,82 & 36,03 & 212,00 & 0,21 & 10,77 \\
\hline
\end{tabular}

$\mathrm{S}$ - superior; $\mathrm{M}$ - médio; I - inferior. Fonte: Autores.

Os resultados mostram um comportamento satisfatório do produto comercial Valio (óleo de laranja) em comparação ao óleo de mineral (Aureo) e ao adjuvante (Vortexy). Os valores observados de diâmetro de gotas, cobertura e número de gotas mostram que o Valio possui um potencial de uso em substituição ao óleo mineral quanto na função de adjuvante em caldas de aplicação de defensivos agrícolas. A cobertura da parte inferior do dossel tem sido baixa na grande parte das aplicações. Bradley et al. (2007) encontraram valores de cobertura das folhas do terço inferior menor que 6\% para tratamentos sem assistência de ar em uma safra com bom fechamento das entrelinhas da cultura da soja e de até $10 \%$ para os mesmos tratamentos em um ano com a soja menor. Já Bretthauer et al. (2008) encontraram valores de cobertura do terço inferior da cultura de no máximo 3,8\% entre os tratamentos avaliado (Figura 1). 
Figura 1. Papeis hidrossensíveis representativos dos diferentes tratamentos.

\begin{tabular}{|c|c|c|}
\hline & io & \\
\hline 16, & 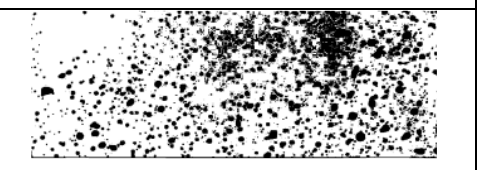 & $\begin{array}{c}4 \\
40\end{array}$ \\
\hline \multicolumn{3}{|c|}{ T1 - Valio ${ }^{\circledR}\left(0,05 \mathrm{~L} 100 \mathrm{~L}\right.$ água $\left.^{-1}\right)$} \\
\hline 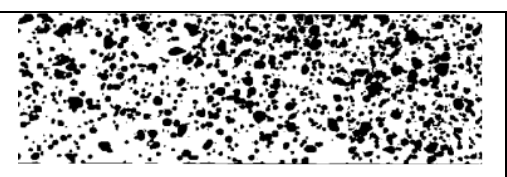 & 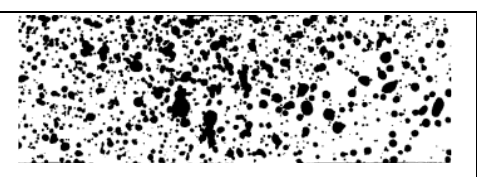 & 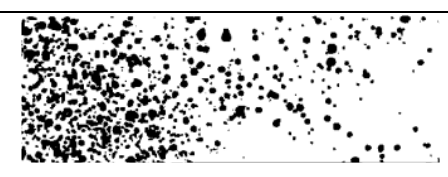 \\
\hline \multicolumn{3}{|c|}{ T1 - Valio ${ }^{\circledR}\left(0,10\right.$ L 100 L água $\left.^{-1}\right)$} \\
\hline 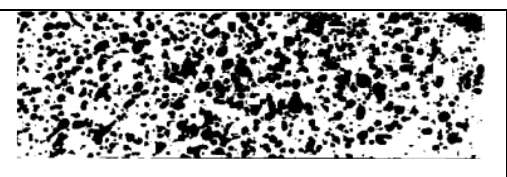 & 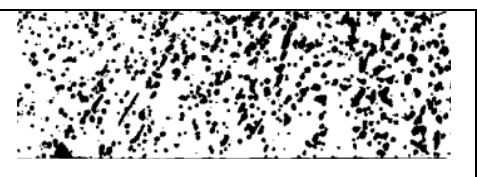 & \\
\hline \multicolumn{3}{|c|}{ T1 - Valio ${ }^{\circledR}\left(0,15\right.$ L 100 L água $\left.^{-1}\right)$} \\
\hline 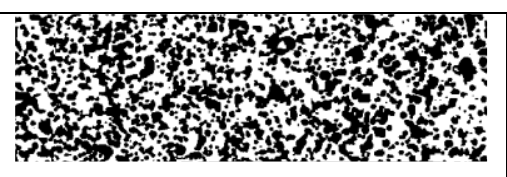 & obstors & ond \\
\hline \multicolumn{3}{|c|}{ T1 - Valio ${ }^{\circledR}\left(0,20\right.$ L 100 L água $\left.^{-1}\right)$} \\
\hline 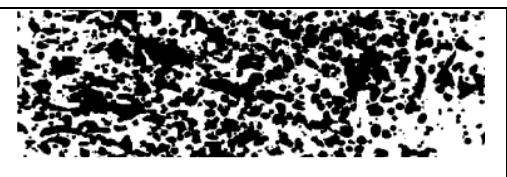 & hothos & \\
\hline \multicolumn{3}{|c|}{ T1 - Valio ${ }^{\circledR}\left(0,25\right.$ L 100 L água $\left.^{-1}\right)$} \\
\hline W & 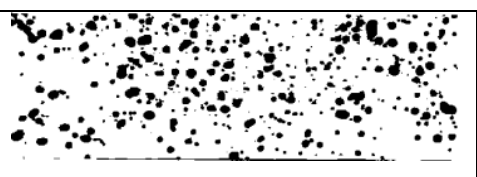 & $a^{2}+4$ \\
\hline \multicolumn{3}{|c|}{ T1 - Vortexy ${ }^{\circledR}\left(0,05 \mathrm{~L} 100 \mathrm{~L}_{\text {água }}{ }^{-1}\right)$} \\
\hline 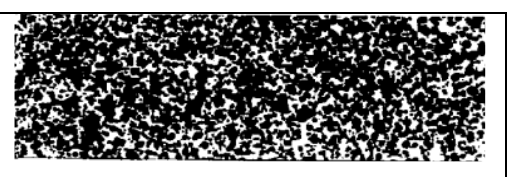 & 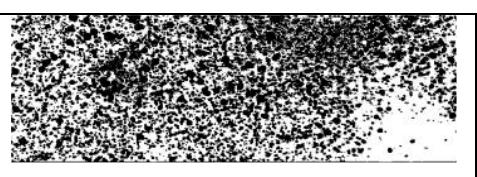 & $\begin{array}{ll}4 \\
40\end{array}$ \\
\hline \multicolumn{3}{|c|}{ T1 - Aureo $^{\circledR}\left(0,25 \mathrm{~L} 100 \mathrm{~L}_{\text {água }}{ }^{-1}\right)$} \\
\hline of & $-4,4)$ & \begin{tabular}{ccc}
$\because$ & 0 \\
\hdashline & $\because$ & $\ddots$
\end{tabular} \\
\hline &, 00 L 100 L água $\left.{ }^{-1}\right)$ & \\
\hline
\end{tabular}

Fonte: Autores.

Todas as doses de Valio testadas apresentaram resultados médios superiores aos observados nos tratamentos Controle. A dose de Valio de $250 \mathrm{~mL} \mathrm{ha}^{-1}$, apresentou os melhores resultados médios dentre as variáveis analisadas. A dose de Valio de $200 \mathrm{~mL} \mathrm{ha}^{-1}$, apresentou resultados equivalentes ao uso de óleo de mineral (Aureo). A dose de $150 \mathrm{~mL} \mathrm{ha}^{-1}$, apresentou resultados que equivalem aos observados no uso do adjuvante (Vortexy). Vale destacar que tanto o óleo mineral (Aureo) 
quanto o adjuvante (Vortexy) apresentaram resultados muito satisfatórios em relação a qualidade de aplicação. O adjuvante (Vortexy) apresentou os melhores resultados de maneira geral quando comparado tanto ao óleo de laranja (Valio) e ao óleo mineral (Aureo) em relação as vaiáveis de qualidade da aplicação realizada.

Resultados obtidos por Nascimento et al. (2012), testando o uso de óleos minerais, vegetais e surfatantes em caldas com fungicida para o controle de ferrugem asiática da soja. Os autores observaram que apesar de os adjuvantes Natur'l Oil, Break Thru e Nimbus apresentarem tendências para maior controle e produtividade, não houve diferenças significativas entre os tratamentos. Os autores observaram diferenças no tamanho de gotas em função do acréscimo dos adjuvantes. Perim (2011), explica que existem duas formas principais, desempenhadas pelos adjuvantes, que resultam no melhor desempenho dos produtos fitossanitários. A primeira pelo aumento da quantidade de ingrediente ativo retido pelo alvo e a segunda a influência positiva sobre sua absorção. Cunha e Perez (2010) observaram que o uso do ajuvante dodecil benzeno, 72,5 g L-1 de N e 46,0 g L-1 de P2O5, aplicado com fungicida para o controle da ferrugem da soja, melhorou o controle da doença, resultando em maior produtividade. Cunha e Perez (2010) observaram que o uso de gotas grossas comparado ao uso de gotas finas, na aplicação de fungicida na soja, reduziu a produtividade da cultura.

\section{Conclusão}

O produto comercial Valio (óleo de laranja) teve um comportamento satisfatório na qualidade da aplicação em comparação ao adjuvante (Vortexy) e óleo mineral (Aureo) utilizados.

Houve aumento no número de gotas de menor diâmetro no terço inferior das plantas, maior cobertura, número de gotas e volume.

A dose de Valio que mais se equiparou ao óleo mineral (áureo) foi de $200 \mathrm{~mL} \mathrm{ha}^{-1}$ e de $150 \mathrm{~mL} \mathrm{ha}^{-1}$ quando comparado ao adjuvante (Vortexy).

\section{Agradecimentos}

Os autores agradecem à Fundação de Amparo à Pesquisa do Brasil (Conselho Nacional de Desenvolvimento Científico e Tecnológico - CNPq), à Coordenação de Aperfeiçoamento de Pessoal de Nível Superior (CAPES); a Fundação de Amparo à Pesquisa do Estado de Goiás (FAPEG); Ministério da Ciência, Tecnologia, Inovações e Comunicações (MCTIC Brasil); a Financiadora de Estudos e Projetos (Finep); Centro de Excelência em Agro Exponencial (CEAGRE); ao Instituto Federal de Educação, Ciência e Tecnologia Goiano (IF Goiano) - Campus Rio Verde; a Universidade de Rio Verde - UniRV; AGIRTEC - Soluções de Precisão; MRE Agropesquisa Ltda., pelo apoio financeiro e estrutural para a realização deste estudo.

\section{Referências}

Araújo, L., Valdebenito-Sanhueza, R. M., \& Stadnik, M. J. (2010). Avaliação de formulações de fosfito de potássio sobre Colletotrichum gloeosporioides in vitro e no controle pós-infeccional da mancha foliar de Glomerella em macieira. Tropical Plant Pathology, 35, 054-059.

Bradley, C. A., Chesrown, C. D., \& Hofman, V. L. (2007). Evaluation of foliar fungicide application methods on soybean. Canadian journal of plant pathology, 29(2), 197-202.

Bretthauer, S. M., Mueller, T. A., Derksen, R. C., Zhu, H., \& Bode, L. E. (2008). The effects of spray application rate and droplet size on applications to control soybean rust. In 2008 Providence, Rhode Island, June 29-July 2, 2008 (p. 1). American Society of Agricultural and Biological Engineers.

Coradini, C., Piccinini, F., Reimche, G. B., Costa, I. F. D. D., \& Machado, S. L. D. O. (2016). Efeito de óleo essencial de laranja associados a fungicidas no controle de doenças foliares do trigo. Summa Phytopathologica, 42, 105-106.

Cunha, J. P. A. R. D., \& Peres, T. C. M. (2010). Influência de pontas de pulverização e adjuvante no controle químico da ferrugem asiática da soja. Acta Scientiarum. Agronomy, 32, 597-602.

da Silva Cavalcante, W. S., da Silva, N. F., Teixeira, M. B., Cabral Filho, F. R., Nascimento, P. E. R., \& Corrêa, F. R. (2020). Eficiência dos bioestimulantes no manejo do déficit hídrico na cultura da soja. IRRIGA, 25(4), 754-763. DOI: https://doi.org/10.15809/irriga.2020v25n4p754-763. 
Research, Society and Development, v. 11, n. 2, e38511225946, 2022

(CC BY 4.0) | ISSN 2525-3409 | DOI: http://dx.doi.org/10.33448/rsd-v11i2.25946

Ferreira, D. F. (2011). Sisvar: a computer statistical analysis system. Ciência e agrotecnologia, 35, 1039-1042.

Iost, C. A., \& Raetano, C. G. (2010). Tensão superficial dinâmica e ângulo de contato de soluções aquosas com surfatantes em superfícies artificiais e naturais. Engenharia Agrícola, 30(4), 670-680.

Jadoski, S. O., Maggi, M. F., Schipanski, C. A., Rezende, J. L., \& Suchoronczek, A. (2011). Efeito de diferentes vazões e adjuvantes na pulverização aérea e terrestre da cultura do milho. Revista Brasileira de Tecnologia Aplicada nas Ciências Agrárias, 2(2), 139-154.

Juliatti, F. C., Juliatti, B. C. M., \& Jaccoud-Filho, D. S. (2013). Technology of pesticide application in corn-nozzles, sprays volume, economic analysis and diseases control. Fungicides-Showcases of Integrated Plant Disease Management from Around the World, 99-114.

Lenz, G., Balardin, R. S., Minuzzi, S. G., Tormen, N. R., \& Marques, L. N. (2011). Espectro de gotas e idade de trifólios na taxa de absorção e efeito residual de fungicidas em soja. Ciência Rural, 41, 1702-1708.

Nascimento, J. M. D., Gavassoni, W. L., Bacchi, L. M. A., Zuntini, B., Mendes, M. P., Leonel, R. K., \& Pontim, B. C. A. (2012). Associação de adjuvantes à picoxistrobina+ ciproconazol no controle da ferrugem asiática da soja. Summa Phytopathologica, 38, $204-210$.

Perim, L. (2011). Efeitos de óleos nas características físicas e químicas da calda de aplicação e na ação da atrazina.Dissertação (Mestrado em Agricultura). Faculdade de Ciências Agronômicas, Universidade Estadual Paulista, Botucatu.

Souza, B. J. R. D., Perez, P. H., Bauer, F. C., Raetano, C. G., Weirich, P. H., \& Garcia, L. C. (2014). Adjuvantes em pulverizações de fungicidas na cultura do trigo. Ciência Rural, 44, 1398-1403.

Theisen, G. (2004). Tecnologia de aplicação de herbicidas: teoria e prática. Aldeia Norte; FUNDACEP.

Tinos, A. C., Compagnon, A. M., Sales, J. G. C., \& Lopes, R. A. P. (2011). Efeito da adição de glicerina como adjuvante à calda de pulverização em aplicação terrestre. Revista Brasileira de Tecnologia Aplicada nas Ciências Agrárias, 3(3).

Tormen, N. R., da Silva, F. D., Debortoli, M. P., Uebel, J. D., Fávera, D. D., \& Balardin, R. S. (2012). Deposição de gotas no dossel e controle químico de Phakopsora pachyrhizi na soja. Revista Brasileira de Engenharia Agrícola e Ambiental, 16, 802-808.

Vargas, L., \& Roman, E. S. (2006). Conceitos e aplicações dos adjuvantes. Embrapa Trigo-Documentos (INFOTECA-E).

Zampiroli, R., de Alvarenga, C. B., Rinaldi, P. C. N., de Carvalho, V. A. M., do Prado, J. R., \& da Silva Neto, A. T. (2019). Parâmetros técnicos da tecnologia de aplicação aplicados à pulverização hidropneumática em diferentes condições operacionais. Revista de Ciências Agrárias Amazonian Journal of Agricultural and Environmental Sciences, 62.

Zhu, H., Salyani, M., \& Fox, R. D. (2011). A portable scanning system for evaluation of spray deposit distribution. Computers and Electronics in Agriculture, 76(1), 38-43. 